\title{
Investigation of predictive potential of TPH1 common polymorphisms in idiopathic scoliosis
}

Svetla Todorova Nikolova ${ }^{1}$, Vasil Tsankov Yablanski², Evgeni Nedev Vlaev², Alexey Slavkov Savov ${ }^{1}$, Ivo Marinov Kremensky ${ }^{3}$

${ }^{1}$ National Genetic Laboratory, Medical University, Sofia, Bulgaria ${ }^{2}$ Department of Orthopedics and Traumatology, Tokuda Hospital Sofia, Bulgaria

${ }^{3}$ Molecular Medicine Center, Medical University, Sofia, Bulgaria

Correspondence: Svetla Nikolova, National Genetic Laboratory, Department of Obstetrics and Gynecology, Faculty of Medicine, Medical University, Bulgaria E-mail: snikolova83@abv.bg

Received: 08 May 2016, Accepted: 10 June 2016 DOI: 10.5799 /jcei.328607

\section{A B S T R A C T}

Objective: The association studies are the predominant type of studies on genetics of the common diseases. The present case-control study aims to investigate the association between the promoter polymorphism TPH1 (rs10488682 T/A) and the predisposition to idiopathic scoliosis (IS) in a Bulgarian population sample.

Methods: A total of 105 patients and 210 healthy gender-matched controls were included. The TPH1 promoter polymorphism was genotyped by amplification followed by restriction. The statistical analysis was performed by the Pearson's chi-squared test and the Fisher's exact test. A value of $p$ less than 0.05 was considered to be statistically significant.

Results: The results indicated that TPH1 (rs10488682) is not associated with the susceptibility to IS, the onset of the disease, the family history or the gender. On the basis of these preliminary results, the examined polymorphic variant could not be considered as a predisposing factor for IS in Bulgarian patients.

Conclusion: Much larger case-control studies will be needed to examine the role of this TPH1 functional genetic variant in the etiology and pathogenesis of IS in Caucasian population. The identification of molecular markers for IS could be useful for early detection of the predisposition among the relatives and for more accurate prognosis of the risk for a rapid progression of the curve in the affected children.

Keywords: idiopathic, scoliosis, TPH1, polymorphism, association

\section{INTRODUCTION}

The association studies are the predominant type of studies on genetics of the common diseases. The study begins with a selection of one or more putative candidate genes based on hypotheses, including biological systems involved in the development of deformity and assumptions based on the results of clinical observations. This is followed by the assessment and selection of polymorphisms, preferably single nucleotide polymorphisms (SNPs). A particular genetic variant is verified for association with a disease by observing its occurrence in random test subjects (cases) and in selected control subjects. Finally, the polymorphic variant could be evaluated for its association with the disease prognosis and diagnosis and its future potential as a biomarker. This makes the knowledge derived from candidate gene studies valuable and clinically relevant as a potential disease diagnostic tool and for personalised medicine initiatives in future treatments of the genetic disorders [1-4].

One of the leading hypotheses for the etiology of idiopathic scoliosis (IS) is the neuroendocrine hypothesis including abnormalities in the melatonin synthesis and signaling pathway [5]. Previous experimental data indicated that some animal models like pinealectomized chicken and melatonin-deficient rats can develop spinal deformities that could be prevented by the melatonin readministration [6-8]. The melatonin 
is a bone protector that may impair the osteoblast and osteoclast activity through different mechanisms [9] and the suppression of the melatonin secretion can decrease the serum calcium concentration, an effect prevented by the melatonin administration [10]. The data regarding melatonin levels in the human deformities are controversial [11] and suggest the role of some functional genetic polymorphisms decreasing the melatonin production, but not leading to real melatonin deficiency, in the etiopathogenesis of IS. The variations in the melatonin levels could increase the susceptibility to deformities.

The gene for tryptophan hydroxylase 1 (TPH1) encodes an enzyme that is essential for the biosynthesis of serotonin - a precursor to melatonin [12]. The promoter polymorphism TPH1 (rs10488682 T/A) can contribute to reduced synthesis of both serotonin and melatonin [13]. Previous studies suggest that the $\mathrm{TPH} 1$ gene is a predisposing factor for IS and there is a relationship between the dyssynthesis of melatonin and adolescent idiopathic scoliosis (AIS) in Chinese population [14].

The present case-control study aims to investigate the association between the promoter polymorphism TPH1 (rs10488682 T/A) and the predisposition to IS in a Bulgarian population sample.

\section{METHODS}

A total of 105 patients and 210 healthy gender-matched controls were included. All participants in the study were informed about its purpose and were included only after the subjects/families signed their informed consent. Peripheral blood samples were obtained from the patients and the control subjects. The study protocol was approved by the University Ethics Committee. This study was performed in accordance with the current (2013) version of Declaration of Helsinki.

\section{Patients}

Patients with IS were recruited with the help of the orthopaedic surgeons. The IS diagnosis was confirmed clinically and radiologically. The secondary scoliosis due to syndromic and nonsyndromic conditions was excluded. The curves were measured by the Cobb method. The mean value of the Cobb angle was $54,6 \pm 22,7^{\circ}$. The mean age at the beginning of the disease was $11.2 \pm 3.1$ years. The cases were separated according to the age of onset of IS in three subgroups: infantiles $(n=4)$, juveniles $(n=23)$ and adolescents $(\mathrm{n}=78)$. In this study, male $(\mathrm{n}=19)$ and female $(\mathrm{n}=86)$ individuals were included. Additionally, patients with positive family history of IS $(n=28)$ and sporadic cases $(n=77)$ were included.

\section{Controls}

The control group including healthy subjects without clinical signs of IS was recruited from a pool of unrelated gender-matched volunteers from hospital units and clinics as well as hospital staff members and students. The controls were selected among adult patients ( $\geq 18$ years old) with skeletal maturity and negative family history of IS. Primary radiological examination was not performed in the control group.

\section{Laboratory methods}

Genomic DNA was extracted from peripheral blood leucocytes by using the magnetic bead technology (chemagic DNA Blood Kit special, Chemagen, Baesweiler, Germany) on an automated high throughput nucleic acid isolation platform (chemagic Magnetic Separation Module I, Chemagen, Baesweiler, Germany).

The TPH1 promoter polymorphism was genotyped by amplification followed by restriction. The primer sets are listed in Table 1.

The polymerase chain reaction (PCR) was carried out in a a reaction mix of $20 \mu$ containing 100 ng DNA, 10X Prime Taq buffer (Genet Bio, Daejeon, Korea), 10 mM dNTPs Mixture (Genet Bio, Daejeon, Korea), 20 pmol Forward and Reverse primers (AlphaDNA, Montreal, Canada), and 0.1 U Prime Taq DNA Polymerase (Genet Bio, Daejeon, Korea). The ampification was performed in an AB 2720 Thermocycler (Life Technologies, NY, USA) with an initial denaturation at $94^{\circ} \mathrm{C}$ for five minutes and a final extension of seven minutes at $72^{\circ} \mathrm{C}$. The following thermal cycle was repeated 30 times: denaturation at $94^{\circ} \mathrm{C}$ for 30 seconds, annealing for 30 seconds at $58^{\circ} \mathrm{C}$, and extension at $72^{\circ} \mathrm{C}$ for 30 seconds.

The restriction fragment length polymorphism (RFLP) analysis was performed with the endonuclease SpeI (NEB, Ipswich, MA, USA) according to the manufacturer's instructions and the restriction fragments were separated on an agarose $3 \%$ gel in a VG-SYS Horizontal Electrophoresis System (Biochrom, Miami, USA). The lengths of the fragments representing the genotypes are presented in Table 2.

Statistical methods. The test for Hardy-Weinberg equilibrium was performed. The Pearson's chi-squared test was used for the genotype and allele comparisons between the cases $\left(n_{1}>30\right)$ and controls $\left(n_{2}>60\right)$ and the Fisher's exact test was used for the smaller subgroups. A value of $\mathrm{p}<0.05$ was considered to be statistically significant in both cases. Additionally, odds ratios (ORs) were calculated with a 95\% confidence interval (95\% CI). The statistical analysis was conducted with the IBM SPSS 19.0 (NY, USA) software package for Windows.

Table 1. PCR primers

\begin{tabular}{lc}
$\begin{array}{l}\text { Gene, } \\
\text { Polymorphism }\end{array}$ & \multicolumn{1}{c}{ Primers } \\
\hline TPH1 & F: 5'-AAGAAGTTGCACAATGCAGACA-3' \\
(rs10488682 T/A) & R: 5'-GTTGGGAAGACTGCAAGAAGC-3' \\
\hline
\end{tabular}

PCR: Polymerase chain reaction; TPH1: Tryptophan hydroxylase 1 


\begin{tabular}{lcccc} 
Table 2. PCR-RFLP protocol & & & \\
\hline $\begin{array}{l}\text { Gene, } \\
\text { Polymorphism }\end{array}$ & Annealing Temperature, ${ }^{\circ} \mathrm{C}$ & $\begin{array}{c}\text { PCR Product } \\
\text { Size, bp }\end{array}$ & Restriction Enzyme & $\begin{array}{c}\text { Restriction } \\
\text { Fragments, bp }\end{array}$ \\
\hline & 58 & & AA: 260 \\
TPH1 (rs10488682 T/A) & & 260 & Spel & TA: $260+146+114$ \\
& & TT: $146+114$ & \\
\hline
\end{tabular}

PCR: Polymerase chain reaction; RFLP: Restriction fragment length polymorphism; TPH1: Tryptophan hydroxylase 1; bp: base pair.

\section{RESULTS}

We separated the cases in subgroups according to age, gender and family history and then investigated the associations in the general sample and in the different subgroups under genotypic (co-dominant, dominant and recessive) and allelic model.

\section{Genotypes were in Hardy-Weinberg equilibrium.}

The overall frequencies of the genotypes and alleles of TPH1 (rs10488682 T/A) in the patients with IS were comparable with the controls (AA vs. TA vs. TT, $p=0.12$ and A vs. T, $p=0.11$ ) but the dominant model was close to a statistically significant result (TT + TA vs. AA, p = 0.05, OR: 1.6, 95\% CI: 0.99-2.57).

The infantile group consisting of 4 patients was not representative and so was not analyzed separately. In the subgroup of adolescents as well as in the subgroup of juveniles, the genotype and allele frequencies of the TPH1 polymorphism were comparable between the cases and the controls $(\mathrm{p}>0.05)$.

The genotype and allele distributions of the TPH1 promoter polymorphism were similar for the familial and the non-familial cases in both subgroups ( $\mathrm{p}>0.05)$.

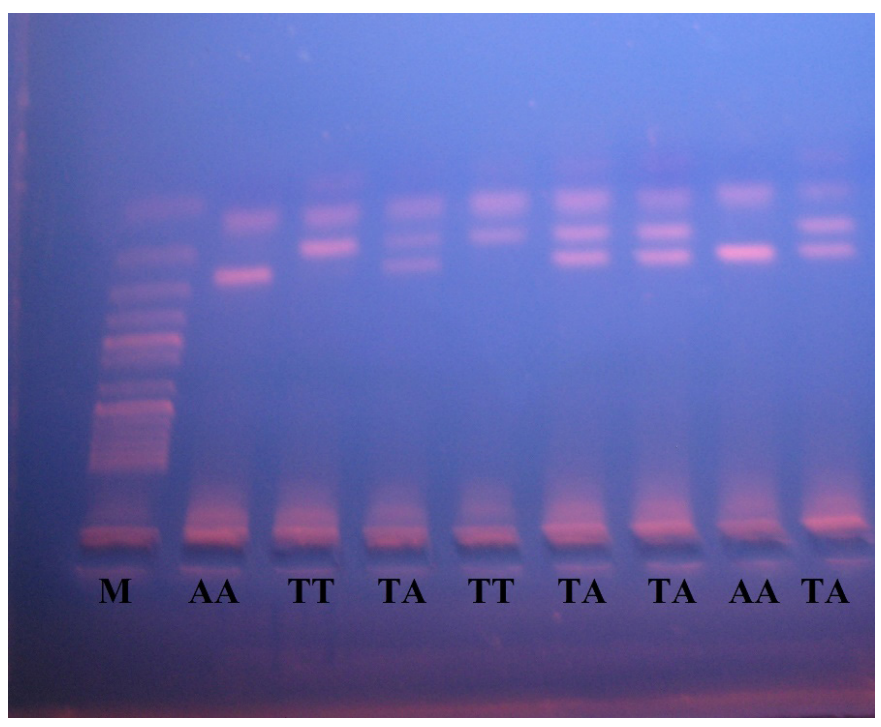

Figure 1. Results from the restriction analysis of TPH1 (rs10488682 T/A): AA - homozygotes (260 bp); TA - heterozygotes (260 + $146+$ $114 \mathrm{bp})$; TT - homozygotes (146 + 114 bp); M - ladder 100 bp. C - negative control.
No statistically significant association between the TPH1 polymorphism and the clinical phenotype was observed for the female and the male patients in both subgroups ( $\mathrm{p}>0.05$ ).

The odds ratios of the genotypes and alleles in the subgroups are summarised in Table 3 .

Sample results from the restriction analysis are presented in Figure 1.

\section{DISCUSSION}

The association study conducted from Wang et al. [2008] in a Chinese population sample was the first study to investigate the possible association between TPH1 and the etiopathogenesis of AIS (103 cases/108 controls). The authors examined a total of 16 SNPs presented in TPH1 and AANAT and found a positive association between TPH1 (rs10488682 T/A) and the susceptibility to AIS. No SNP was found to be correlated with the gender or the Cobb angle of the patients [14].

Later, TPH1 (rs10488682 T/A) was found to be not associated with the occurrence or progression of AIS in a larger study in Japanese population (798 cases/1239 controls) [15].

An American cohort study (589 cases/1533 controls) examined 4 SNPs in the TPH1 gene but no statistically significant association with AIS was found [16].

All the studies included only patients with AIS (late onset IS) that is the most common [17]. There is a need for studies of the early onset IS (infantile and juvenile). It is possible the participation of different genetic variants in the etiopathogenesis of the early and late onset scoliosis [18]. In our study, we selected the cases among patients with infantile, juvenile and adolescent IS and then we investigated the associations in the general sample and in the different subgroups.

In the general sample, the genotype and allele frequencies were comparable between the cases and the controls, and hence TPH1 (rs10488682 T/A) could not be considered as a susceptibility factor for IS in Bulgarian patients. The major limitation of the current study is the small sample sizes that could affect the statistical power of the results. As an example, for the dominant genetic model (TT+TA vs. AA) the estimated statistical power is $62.2 \%$. This means that we would need a sample of 228 per group to yield power of $80 \%$. In other words, the patient group must be twice larger. 
Nikolova ST, et al. TPH1 common polymorphisms in idiopathic scoliosis

Table 3. Odds ratios of genotypes and alleles of tryptophan hydroxylase 1 (TPH1) gene in different subgroups with idiopathic scoliosis (IS)

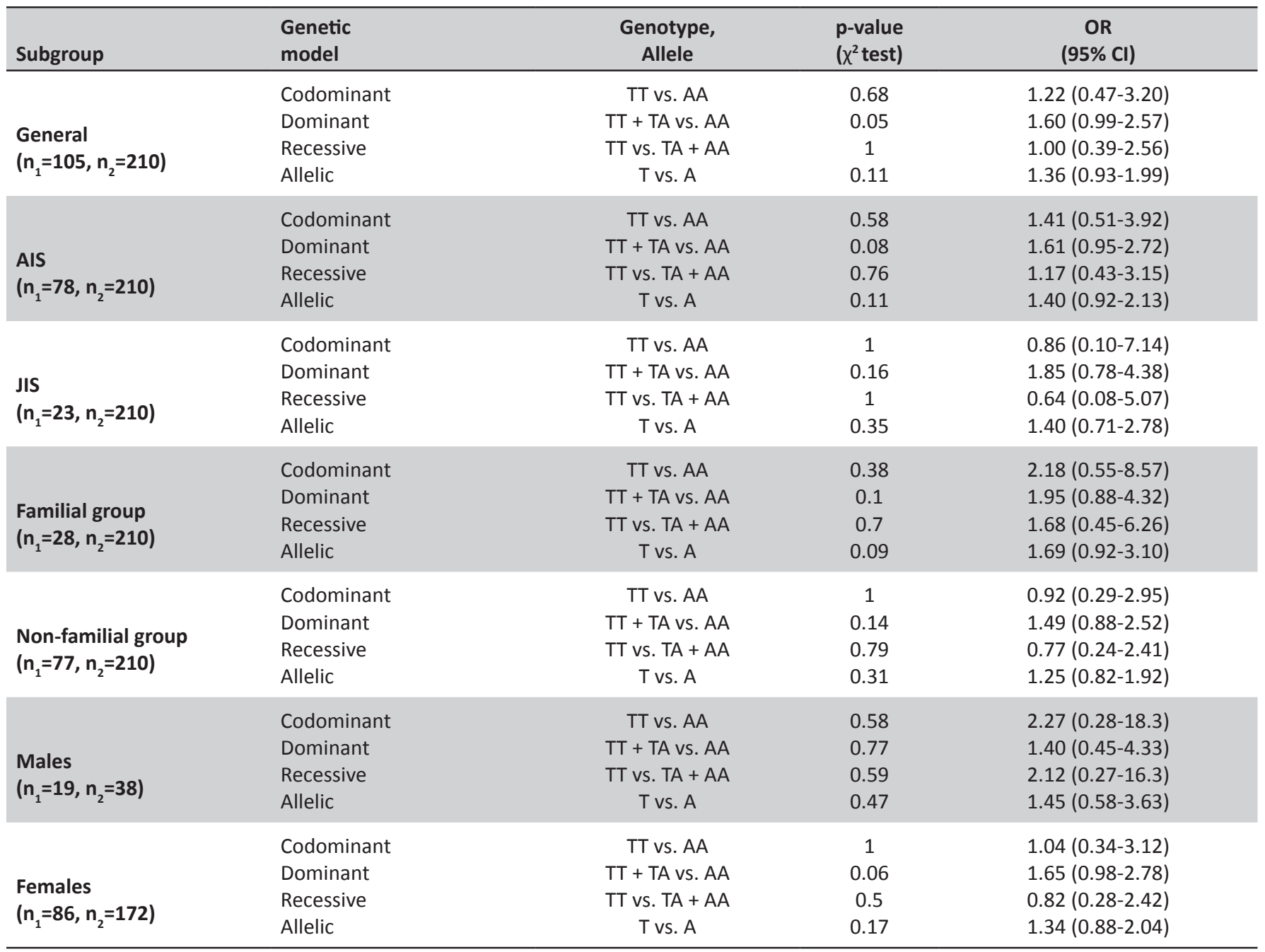

A value of $p<0.05$ was considered to be statistically significant. AIS: Adolescent idiopathic scoliosis; JIS: Juvenile idiopathic scoliosis; OR: Odds ratio; CI: confidence interval

In the AIS subgroup, the genotype and allele frequencies were also comparable between the cases and the controls, and hence TPH1 (rs10488682) could not be associated with the late onset IS. These results correspond to those obtained previously in Caucasian population [16]. Extended case-control studies will be necessary to examine the role of this TPH1 genetic variant in the etiology and pathogenesis of the early onset IS in different population groups.

We selected the cases among patients with positive family history and negative family history as well. We assumed that it is possible the participation of different genetic variants in the etiopathogenesis of the sporadic and the familial cases. On the basis of the present results, the genotypes and the alleles of TPH1 (rs10488682) could not be associated with the familial history of IS. Replication case-control studies will be necessary to examine the role of the TPH1 gene in the etiopathogenesis of the familial and sporadic IS in the different population groups.
Scoliosis is more common in the females than the males [19]. It is possible the participation of different genetic variants in the etiopathogenesis of IS in the male and the female patients. The obtained results showed that the genotypes and alleles of TPH1 ( rs10488682 T/A) could not be associated with the gender of the patients.

In conclusion, the results indicated that TPH1 (rs10488682) is not associated with the susceptibility to IS, the onset of the disease, the family history or the gender.

On the basis of these preliminary results, the examined polymorphic variant TPH1 (rs10488682) could not be considered as a predisposing factor for IS or AIS in Bulgarian patients.

Much larger case-control studies will be needed to examine the role of this TPH1 functional genetic variant in the etiology and pathogenesis of IS in Caucasian population. 
The data regarding the association between the primary scoliosis and the human MTNR1B genetic variants $[15,16,19-$ 23] do not exclude the role of other components of the melatonin production and the melatonin signaling pathway in the IS etiopathogenesis.

The identification of molecular markers for IS could be useful for early detection of the predisposition among the relatives and for more accurate prognosis of the risk for a rapid progression of the curve in the affected children. That will permit prophylaxis and early treatment with less invasive procedures.

\section{Acknowledgments}

This manuscript does not include any information about medical device (s) or drug (s). No benefits in any form have been or will be received from any commercial entity related directly or indirectly to the subject of this manuscript. We would like to thank all participants for their support in this study.

Declaration of conflicting interests: The authors declare that they have no conflict of interest.

Financial Disclosure: No financial support was received

\section{REFERENCES}

1. Patnala R, Clements J, Batra J. Candidate gene association studies: a comprehensive guide to useful in silico tools. BMC Genetics. 2013;14:39.

2. Kwon JM, Goate AM. The candidate gene approach. Alcohol Res Health. 2000,24:164-8.

3. Collins FS, Guyer MS, Chakravarti A: Variations on a theme: cataloging human DNA sequence variation. Science. 1997,278:1580-1.

4. Peters BJM, Rodin AS, De Boer A, Maitland-van der Zee A-H. Methodological and statistical issues in pharmacogenomics. J Pharm Pharmacol. 2010,62:161-6.

5. Moreau A, Wang DS, Forget S, et al. Melatonin signaling dysfunction in adolescent idiopathic scoliosis. Spine. 2004;29:1772-81.

6. Machida M, Dubousset J, Imamura Y, Iwaya T, Yamada T, Kimura J, et al. Pathogenesis of idiopathic scoliosis: sEPs in chicken with experimentally induced scoliosis and in patients with idiopathic scoliosis. J Pediatr Orthop. 1994;14:329-35.

7. Machida M, Dubousset J, Imamura Y, Iwaya T, Yamada T, Kimura J. Role of melatonin deficiency in the development of scoliosis in pinealectomized chickens. J Bone Joint Surg Br. 1995;77:134-8.

8. Kono H, Machida M, Saito M, et al. Mechanism of osteoporosis in adolescent idiopathic scoliosis: experimental scoliosis in pinealectomized chickens. J Pineal Res. 2011;51:387-93.
9. Cardinali D, Ladizesky M, Boggio V, Cutrera R, Mautalen EC. Melatonin Use as a Bone-Protecting Substance. In: Pandi-Perumal SR, Cardinali DP, editor. Melatonin: Biological Basis of its Function in Health and Disease. 2004

10. Grivas TB, Savvidou OD. Melatonin the "light of night" in human biology and adolescent idiopathic scoliosis. Scoliosis. 2007;2:6.

11. Girardo M, Bettini N, Dema E, Cervellati S. The role of melatonin in the pathogenesis of adolescent idiopathic scoliosis (AIS). Eur Spine J. 2011;20:S68-S74.

12. Gorman KF, Julien C, Moreau A. The genetic epidemiology of idiopathic scoliosis. Eur Spine J. 2012;21:1905-19.

13. Burger EL, Noshchenko A, Patel VV, Lindley EM, Bradford AP. Ultrastructure of Intervertebral Disc and Vertebra-Disc Junctions Zones as a Link in Etiopathogenesis of Idiopathic Scoliosis. Adv Orthop Surg. 2014;2014:850594.

14. Wang H, Wu Z, Zhuang Q, Fei Q, Zhang J, Liu Y, et al. Association study of tryptophan hydroxylase 1 and arylalkylamine $\mathrm{N}$-acetyltransferase polymorphisms with adolescent idiopathic scoliosis in Han Chinese. Spine (Phila Pa 1976). 2008;33:2199-203.

15. Takahashi Y., M. Matsumoto, T. Karasugi, K. Watanabe, K. Chiba, N. Kawakami, et al. Lack of association between adolescent idiopathic scoliosis and previously reported single nucleotide polymorphisms in MATN-1, MTNR1B, TPH1, and IGF1 in a Japanese population. J Orthop Res. 2011;29:1055-8.

16. Nelson LM, Ward K, Ogilvie JW. Genetic variants in melatonin synthesis and signaling pathway are not associated with adolescent idiopathic scoliosis. Spine (Phila Pa 1976). 2011;36:37-40.

17. Altaf F. Adolescent Idiopathic Scoliosis. BMJ. 2013;346:f2508.

18. Ogilvie JW. Update on prognostic genetic testing in adolescent idiopathic scoliosis (AIS). J Pediatr Orthop. 2011;31:S46-S48.

19. Qiu XS, Tang NL, Yeung HY, Qiu Y, Qin L, Lee KM, et al. The role of melatonin receptor 1B gene (MTNR1B) in adolescent idiopathic scoliosis-a genetic association study. Stud Health Technol Inform. 2006; 123:3-8

20. Qiu XS, Tang NL, Yeung HY, Lee KM, Hung VW, Ng BK, et al. Melatonin receptor 1B (MTNR1B) gene polymorphism is associated with the occurrence of adolescent idiopathic scoliosis. Spine (Phila $\mathrm{Pa}$ 1976). 2007;32:1748-53.

21. Shyy W, Wang K, Gurnett CA, Dobbs MB, Miller NH, Wise C, et al. Evaluation of GPR50, hMel-1B, and ROR-alpha melatonin-related receptors and the etiology of adolescent idiopathic scoliosis. J Pediatr Orthop. 2010;3:539-43.

22. Morocz M, Czibula A, Grozer ZB, Szecsenyi A, Almos PZ, Rasko I, et al. Association study of BMP4, IL6, Leptin, MMP3, and MTNR1B gene promoter polymorphisms and adolescent idiopathic scoliosis. Spine. 2011;36:E123-E130.

23. Nikolova S, Yablanski V, Vlaev E, Savov A, Kremensky I. Association study between idiopathic scoliosis and MTNR1B and CHD7 gene polymorphisms in Bulgarian patients. Science \& Technologies: Medicine. 2015;5:81-6. 